\title{
Helicobacter pylori em crianças e associação de cepas CagA na transmissão mãe-filho na Amazônia brasileira
}

\author{
Helicobacter pylori in children and association with CagA strains \\ in mother-child transmission in the Brazilian Amazon region
}

\author{
Vivian D’Annibale Cartágenes ${ }^{1}$, Luísa Carício Martins², Lígia Maia Carneiro ${ }^{3}$, \\ Katarine Antonia dos Santos Barile ${ }^{3}$ Tereza Cristina Corvelo ${ }^{3}$
}

\begin{abstract}
RESUMO
Investigou-se a prevalência de infecção pela Helicobacter pylori em amostras de sangue de 100 crianças de 1 a 12 anos e de suas mães através dos métodos de hemaglutinação indireta e anti-CagA pelo ensaio ELISA. Destas 100 crianças, foram obtidas 79 amostras de fezes e realizada pesquisa de antígenos da bactéria nas fezes por ELISA de captura. Os antígenos foram detectados em 54,4\% (43/79) das crianças, e os anticorpos no soro em 43\% (34/79), métodos que apresentaram desempenhos semelhantes, com maiores discordâncias nas crianças de 1 a 4 anos. A soroprevalência nas crianças foi de 50\% (50/100) e nas mães de 86\% (86/100). Mães infectadas representaram fator de risco 19 vezes superior ao de mães soronegativas para determinar infecção em seus filhos ( $\mathrm{p}<0,05)$, sobretudo as mães com cepas CagA+ $(\mathrm{p}<0,05)$. 0 contato direto pessoa-pessoa pode ser um modo de transmissão desta infecção.
\end{abstract}

Palavras-chaves: Helicobacter pylori. Prevalência. Cepas CagA. Transmissão mãe-filho. Brasil.

\begin{abstract}
The prevalence of Helicobacter pylori infection was investigated in blood samples from 100 children aged 1 to 12 years and from their mothers, by means of the indirect hemagglutination and anti-CagA methods, using ELISA assays. From these 100 children, 79 stool samples were obtained and bacterial antigens in the stools were investigated using capture ELISA. The antigens were detected in 54.4\% (43/79) of the children, and serum antibodies in 43\% (34/79). These methods presented similar performance, with greatest disagreement among the children aged 1 to 4 years. The seroprevalence was 50\% (50/100) among the children and 86\% (86/100) among the mothers. Infected mothers represented a risk factor that was 19 times greater than that of seronegative mothers, with regard to infecting their children $(\mathrm{p}<0.05)$, especially the mothers with CagA+ strains $(\mathrm{p}<0.05)$. Direct person-to-person contact may be a transmission method for this infection.
\end{abstract}

Key-words: Helicobacter pylori. Prevalence. CagA strains. Mother-child transmission. Brazil.

A Helicobacter pylori é uma bactéria Gram-negativa, microaerófila, cujo principal reservatório é o estômago de seres humanos, onde determina inflamação aguda e crônica e, quando a infecção não é tratada, permanece para sempre no hospedeiro, sendo raramente eliminada de forma espontânea ${ }^{34}$. Essa infecção representa um sério problema de saúde pública, pois pode evoluir com graves lesões precursoras de neoplasias nos indivíduos infectados ${ }^{110}$. Apresenta distribuição mundial, mas com prevalências maiores em países em desenvolvimento, relacionadas a fatores contextuais, tais como as precárias condições socioeconômicas e sanitárias dessas regiões ${ }^{20}$. Outras condições também parecem influenciar sua prevalência, como a idade ${ }^{33}$, fatores genéticos do hospedeiro ${ }^{9}$ e da bactéria ${ }^{8}$,

\footnotetext{
1. Núcleo de Vigilância Epidemiológica, Hospital Universitário João de Barros Barreto, Universidade Federal do Pará, Belém, PA. 2. Núcleo de Medicina Tropical, Universidade Federal do Pará, Belém, PA. 3. Laboratório de Imunogenética, Departamento de Genética, Centro de Ciências Biológicas, Universidade Federal do Pará, Belém, PA.

Endereço para correspondência: Dra. Vivian D'Annibale Cartágenes. Rua dos Tamoios 1434/1201, 66025-125 Belém, PA.

Telefax: 559132016699

e-mail: vivi_aslan@yahoo.com.br

Recebido para publicação em 29/12/2009

Aceito em 12/05/2009
}

ou mesmo os fatores ambientais, como hábitos alimentares ${ }^{19}$, consumo de bebidas alcoólicas e fumo ${ }^{4}$.

A bactéria dispõe de genes específicos responsáveis pela sua virulência e patogenicidade, que codificam a citotoxina associada ao gene cagA (CagA), encontrado em aproximadamente $60 \%$ das cepas. Esta proteína bacteriana, altamente imunogênica é injetada no citoplasma da célula hospedeira e exerce um importante papel na indução de sinalização intracelular, promovendo múltiplas mudanças como a ativação da inflamação, conduzindo a severos danos à mucosa gástrica e duodenal, e à produção de anticorpos $\operatorname{anti-CagA}^{810} 18$.

Na maioria dos casos, a infecção é adquirida na infância ${ }^{5}$ ocorrendo um rápido aumento da taxa de prevalência, particularmente nos cinco primeiros anos de vida. É universalmente aceito que a bactéria apenas consiga alcançar a mucosa gástrica através da boca, pois se trata de um microorganismo não invasivo, que parece ser transmitido de pessoa a pessoa de forma direta ou indireta $^{11}$. As principais rotas de transmissão são oral-oral, fecaloral e gastro-oral, pois antígenos da bactéria têm sido detectados na cavidade oral ${ }^{32}$, eliminados através das fezes ${ }^{27}$, como também em conteúdos de vômitos ${ }^{21}$. A concordância de tipos moleculares desta bactéria, entre indivíduos da mesma família, indica que a 
transmissão ocorre entre seus membros ${ }^{11121732}$. A presença de infecção nos pais, especialmente nas mães, é um importante fator de risco para os filhos devido aos contatos provenientes de seus cuidados $^{151732}$. Assim, é possível que as crianças, além de serem mais predispostas a adquirir a infecção por meio de seus hábitos, tenham também a facilidade de transmiti-la, tanto para adultos como para outras crianças, fato que poderia explicar a prevalência intrafamiliar $^{420}$. Considerando a importância da transmissão intrafamiliar na infecção pela Helicobacter pylori, investigou-se sua prevalência e a existência de cepas virulentas CagA entre as crianças e suas respectivas mães, procedentes do Estado do Pará, região norte do Brasil.

\section{MATERIAL E MÉTODOS}

Este estudo descritivo e analítico, do tipo transversal, incluiu 100 binômios mães-filhos, cujas crianças estavam na faixa etária entre 1 e 12 anos e foram atendidas na enfermaria do Hospital Universitário João de Barros Barreto (HUJBB), em Belém, Pará, Brasil, nos períodos de fevereiro a abril e de agosto a setembro de 2001. Foram incluídas crianças com qualquer diagnóstico médico, exceto as que estavam em estado grave, como em instabilidade hemodinâmica e/ou sob cuidados intensivos. Além disso, as crianças deveriam estar acompanhadas pelas suas respectivas mães biológicas, desde que residissem com ela desde o nascimento, e que as mães consentissem em suas participações neste estudo. 0 projeto desta pesquisa foi aprovado pelo Comitê de Ética em Pesquisa com Seres Humanos do Núcleo de Medicina Tropical da Universidade Federal do Pará (UFPA). Um questionário padrão foi aplicado para obtenção de informações relativas às mães e às crianças, com respostas fechadas, para facilitar a codificação dos dados. Os materiais biológicos estudados incluíram amostras de sangue das crianças e de suas mães para a pesquisa de anticorpos contra a bactéria e seu fator de virulência CagA, e de fezes das crianças para a detecção de antígenos específicos da Helicobacter pylori. Uma amostra de $4 \mathrm{~mL}$ de sangue de cada mãe e de seu filho foi obtida através de punção venosa periférica. 0 sangue colhido foi centrifugado a 3.000rotações/min durante 20 minutos, e o soro foi separado e estocado a $-20^{\circ} \mathrm{C}$. As amostras de fezes foram coletadas pelas mães conforme orientação prévia, sendo que as mesmas foram mantidas sob refrigeração por um tempo máximo de duas horas, e posteriormente congeladas a $-20^{\circ} \mathrm{C}$ até serem analisadas.

Para a detecção de anticorpos IgG Helicobacter pylori específicos empregou-se o método de hemaglutinação indireta através de um kit comercial da Viva Diagnostika, Alemanha (Lote: 010206), que tem caráter qualitativo. Para a detecção de anticorpos IgG anti-CagA, foi utilizado o kit comercial Helicobacter P-120 EIA, da VIVA Diagnostica, Hürth, Alemanha (Lote: 001111), que é um ensaio imunoenzimático baseado nos antígenos recombinantes CagA Helicobacter pylori. Para a detecção de antígenos da Helicobacter pylori nas fezes, foi utilizado um kit comercial de ELISA de captura (Platinum HpSA, Meridian Diagnostics, Cincinnati, OH, USA; Lote: 601348.063), que detecta antígenos através de anticorpos policlonais antiHelicobacter pylori. Este é um método qualitativo, altamente sensível (92\% a 94\%) e específico $(92,8 \% \text { a } 95,4 \%)^{3}$. Todos os testes aplicados seguiram as recomendações dos fabricantes.

As informações foram inseridas em um banco de dados e analisadas usando o programa de computação BioEstat 5.02. As informações relativas à infecção pela Helicobacter pylori foram submetidas a uma análise univariada, testando-se a existência de associação entre variáveis independentes (fatores de risco) e a presença de marcadores sorológicos nas crianças e suas mães, com aplicação dos testes qui-quadrado $\left(\chi^{2}\right)$, teste $\mathrm{G}$ e teste exato de Fisher. Os resultados dos métodos diagnósticos no soro e nas fezes das crianças foram analisados pelo teste de McNemar. O limite de significância estatística adotado neste estudo foi $\leq 0.05$.

\section{RESULTADOS}

No grupo das 100 crianças estudadas, 50\% (50) apresentaram anticorpos (Ac) IgG anti-Helicobacter pylori. 0 intervalo de confiança (IC) foi de 40,2 a 59,8. Entre as 79 crianças, que contribuíram com amostras fecais, 54,4\% (43; IC= 43,0 - 65,0) eliminavam antígenos (Ag) específicos da bactéria.

$\mathrm{Na}$ análise dos resultados obtidos em relação à distribuição da infecção pela Helicobacter pylori, nas crianças através da pesquisa de Ac no soro e de Ag nas fezes, a partir das 79 amostras pareadas de soro e fezes, não foram observadas discordâncias estatísticas significativas $\left(\chi^{2}(\mathrm{McNemar} \mathrm{B} / \mathrm{C})=3,047, \mathrm{p}=0,080\right)$. Entretanto, quando estes resultados referentes à infecção pela Helicobacter pylori nas crianças foram distribuídos de acordo com a faixa etária, verificou-se a ocorrência de discordância estatisticamente significativa entre estes testes na faixa etária de 1 a 4 anos, na qual 24,4\% (10/41) apresentaram-se sem Ac no soro, mas com Ag nas fezes. Porém, atingiu 9,5\% (2/21) na faixa etária de 5 a 8 anos e 17,6\% (3/17) na faixa etária de 9 a 12 anos. Obteve-se uma tendência crescente de crianças com Ag nas fezes e Ac no soro conforme o aumento da faixa etária (Tabela 1).

Dentre os 57 meninos, 45,6\% (26) apresentaram sorologia positiva, e dentre as 43 meninas, 55,8\% (24) foram soropositivas. Da mesmaforma, não foram observadas diferenças estatisticamente significativas no estudo da soroprevalência da infecção pela Helicobacter pylori associada com etnia, dieta e higiene bucal, porém, existiu uma associação significativa quando associada ao ato de freqüentar creche ou escola (Tabela 2).

Observou-se que, dentre as 100 mães estudadas, 86\% (86; $\mathrm{IC}=79,2-92,8)$ foram soropositivas para a infecção, sendo que destas 58,1\% (50) apresentavam filhos com a infecção, de modo que o risco de uma criança ser infectada pela Helicobacter pylori quando a mãe apresentou a bactéria foi 19 vezes superior ao de uma criança com mãe soronegativa $(O R=19,444)$, com diferenças estatisticamente significantes. Nota-se que, entre as 39 crianças $\mathrm{CagA}^{+}, 87,2 \%$ (34) possuíam mães também com cepa $\mathrm{CagA}^{+}$, enquanto entre as 11 crianças negativas para esta cepa, 81,8\% (9) possuíam mães com cepa $\mathrm{CagA}^{+}$, de modo que a soroprevalência para cepas virulentas $\mathrm{CagA}^{+}$entre crianças foi maior (78\%; 39/50) do que as CagA . Por outro lado, todas as 14 mães que tinham sorologia negativa para a infecção pela Helicobacter pylori possuíam filhos também negativos (Tabela 3). 
TABELA 1

Distribuição da infecção pela Helicobacter pylori nas crianças de acordo com a faixa etária e a presença de anticorpos no soro e de antígenos nas fezes.

\begin{tabular}{|c|c|c|c|c|c|c|c|c|c|c|}
\hline \multirow{3}{*}{ Faixa etária (anos) } & \multicolumn{8}{|c|}{ Anticorpos IgG no soro e antígenos nas fezes das crianças } & & \\
\hline & \multicolumn{2}{|c|}{$\mathrm{Ac}+/ \mathrm{Ag}+$} & \multicolumn{2}{|c|}{$\mathrm{Ac}+/ \mathrm{Ag}-$} & \multicolumn{2}{|c|}{$\mathrm{Ac}-/ \mathrm{Ag}+$} & \multicolumn{2}{|c|}{ Ac-/Ag- } & \multicolumn{2}{|c|}{ Total } \\
\hline & $\mathrm{n}^{0}$ & $\%$ & $\mathrm{n}^{\mathrm{o}}$ & $\%$ & $\mathrm{n}^{\mathrm{o}}$ & $\%$ & $\mathrm{n}^{\mathrm{o}}$ & $\%$ & $\mathrm{n}^{0}$ & $\%$ \\
\hline $1 \mathrm{a} 4$ & 4 & 9,8 & 3 & 7,3 & 10 & 24,4 & 24 & 58,5 & 41 & 100,0 \\
\hline 5 a 8 & 14 & 66,7 & 2 & 9,5 & 2 & 9,5 & 3 & 14,3 & 21 & 100,0 \\
\hline 9 a 12 & 10 & 58,8 & 1 & 5,9 & 3 & 17,6 & 3 & 17,6 & 17 & 100,0 \\
\hline Total & 28 & 35,4 & 6 & 7,6 & 15 & 19,0 & 30 & 38,0 & 79 & 100,0 \\
\hline
\end{tabular}

+: presentes. -: ausentes. $G_{(6 G \mathrm{~L})}$ Williams corrigido $=27,706, \mathrm{p}=0,000$.

TABELA 2

Soroprevalência da infecção pela Helicobacter pylori nas crianças de acordo com ato de freqüentar creche ou escola.

\begin{tabular}{|c|c|c|c|c|c|c|}
\hline \multirow[b]{3}{*}{ Freqüenta creche ou escola } & \multicolumn{4}{|c|}{ Anticorpos IgG no soro das crianças } & & \\
\hline & \multicolumn{2}{|c|}{ presente } & \multicolumn{2}{|c|}{ ausente } & \multicolumn{2}{|c|}{ Total } \\
\hline & $\mathrm{n}^{0}$ & $\%$ & $\mathrm{n}^{0}$ & $\%$ & $\mathrm{n}^{\mathrm{o}}$ & $\%$ \\
\hline$\overline{\mathrm{Sim}}$ & 39 & 67,2 & 19 & 32,8 & 58 & 100,0 \\
\hline Não & 11 & 26,2 & 31 & 73,8 & 42 & 100,0 \\
\hline Total & 50 & 50,0 & 50 & 50,0 & 100 & 100,0 \\
\hline
\end{tabular}

TABELA 3

Distribuição da soroprevalência para a infecção pela Helicobacter pylori entre as crianças e suas mães de acordo com cepas CagA.

\begin{tabular}{|c|c|c|c|c|c|c|c|c|}
\hline \multirow[b]{3}{*}{ Anticorpos IgG no soro das crianças } & \multicolumn{6}{|c|}{ Anticorpos IgG no soro das mães } & & \\
\hline & \multicolumn{2}{|c|}{$\mathrm{Hp}+\mathrm{CagA}+{ }^{1}$} & \multicolumn{2}{|c|}{$\mathrm{Hp}+\mathrm{CagA}^{2}$} & \multicolumn{2}{|c|}{ Hp- CagA- ${ }^{3}$} & \multicolumn{2}{|c|}{ Total } \\
\hline & $n^{0}$ & $\%$ & $\mathrm{n}^{\circ}$ & $\%$ & $\mathrm{n}^{0}$ & $\%$ & $\mathrm{n}^{0}$ & $\%$ \\
\hline $\mathrm{Hp}+\mathrm{CagA}+{ }^{1}$ & 34 & 87,2 & 5 & 12,8 & - & - & 39 & 100,0 \\
\hline $\mathrm{Hp}+\mathrm{CagA}^{2}$ & 9 & 81,8 & 2 & 18,2 & - & - & 11 & 100,0 \\
\hline Hp- CagA- 3 & 28 & 56,0 & 8 & 16,0 & 14 & 28,0 & 50 & 100,0 \\
\hline Total & 71 & 71,0 & 15 & 15,0 & 14 & 14,0 & 100 & 100,0 \\
\hline
\end{tabular}

${ }^{1}$ Sorologia positiva para a cepa CagA. ${ }^{2}$ Sorologia positiva para a infecção e negativa para a cepa CagA. ${ }^{3}$ Sorologia negativa para a infecção.

$\mathrm{G}_{(4 \mathrm{GL})}$ Williams corrigido $=14,891, \mathrm{p}=0,004$.

\section{DISCUSSÃ̃o}

No presente estudo, identificou-se uma soroprevalência da infecção em 50\% das crianças investigadas (IC= 40,2 - 59,8), sendo que estas foram provenientes de mães infectadas. Estes dados foram concordantes com outros estudos de diversas partes do Brasil ${ }^{25} 2930$ e do mundo ${ }^{3322}$, reforçando a hipótese de uma transmissão familiar.

Tem sido descrito que o teste ELISA nas fezes apresenta sensibilidade e especificidade mais elevadas em comparação aos testes sorológicos ${ }^{34}$. Neste estudo, não foram observadas discordâncias estatisticamente significativas entre estes resultados, quando as 79 amostras pareadas de fezes e soro das crianças foram relacionadas sem distinção de faixa etária. Por outro lado, na análise destes resultados distribuídos por faixa etária, verificou-se que estas proporções diferiam estatisticamente, principalmente no intervalo de 1 a 4 anos. Tal discordância ocorreu pela presença de antígenos nas fezes com ausência de anticorpos Helicobacter pylori específicos, o que sugere uma aquisição da infecção recente, ou anticorpos em níveis ainda não detectáveis. A presença de anticorpos no soro, sem antígenos nas fezes, pode representar o desaparecimento da infecção, uma vez que o teste sorológico empregado nesta pesquisa indica unicamente uma exposição prévia ao microrganismo, mas não discrimina indivíduos sadios ou com infecção ativa ${ }^{2434}$. Já o teste nas fezes pode ser usado para o diagnóstico inicial da infecção, quando o indivíduo ainda não apresenta níveis de anticorpos detectáveis, bem como para confirmar a erradicação da Helicobacter pylori após quatro semanas do término do tratamento, quando ainda são verificados anticorpos no soro ${ }^{24}$. 0 imunoensaio nas fezes foi aprovado pela Food and DrugAdministration desde 1998, tanto para o diagnóstico como para o controle da terapia, inclusive em crianças ${ }^{24} 34$. Embora a sorologia seja um teste pouco oneroso e de fácil execução, a pesquisa de antígenos nas fezes tem a vantagem de ser não invasivo, fácil e rápido de operar, e apenas uma amostra de 
fezes é necessária ${ }^{34}$. Além disto, também é mais econômico que o teste respiratório com carbono marcado (C-UBT), fatos que conduziram o European Helicobacter pylori Study Group a indicá-lo como uma alternativa ao teste respiratório ${ }^{3}$.

A infância, apesar de ser o período descrito em outros estudos como o de maior incidência de infecção pela Helicobacter pylori $^{26}$, é também o de maior perda da infecção primária com posterior possibilidade de reinfecção. Sabe-se, também, que ocorre um decréscimo lento de títulos de anticorpos após a perda da infecção, espontânea ou após uso de antibióticos ${ }^{1834}$.

A soroprevalência encontrada dentre todas as mães foi de $86 \%$. Esta taxa foi elevada, e pode ser considerada similar a de outros países em desenvolvimento, como Quênia ${ }^{22}$, Colômbia ${ }^{16}$, Peru, Gâmbia e Índia ${ }^{36}$.

Ainda não está definida qual a rota predominante de aquisição da Helicobacter pylori. As variáveis que envolvem as rotas oraloral, fecal-oral e gastro-oral estão proximamente interligadas, fato que dificulta a identificação clara da forma predominante de transmissão da Helicobacter pylori nas populações. Estas poderiam ser rotas de infecção primária em crianças e de reinfecção em crianças e adultos ${ }^{2021}$. A transmissão oral-oral ocorre em determinadas situações que incluem, sobretudo, os hábitos dos indivíduos e o maior contato íntimo entre eles. A bactéria, presente no estômago, alcança a cavidade oral através do refluxo gastroesofágico e coloniza-se na boca, particularmente na placa dental e na língua. A saliva pode ser um veículo de transmissão para outros indivíduos ${ }^{713}$. Em ambiente onde a higiene é precária, a rota fecal-oral também pode estar favorecida $a^{13}$. A condução da bactéria do estômago ao intestino, a partir da constante produção de muco e suco gástrico, pode favorecer sua transferência e eliminação através das fezes ${ }^{27}$, contaminando a água ${ }^{6}$ e/ou alimentos ${ }^{11}$. Entre indivíduos do ambiente familiar ou que convivem juntos em escolas, creches e outras instituições, as rotas fecal-oral e oral-oral podem atuar simultaneamente, incluindo ainda a gastro-oral, principalmente na presença de crianças, nas quais os vômitos são normalmente mais comuns ${ }^{2133}$. A bactéria, que é encontrada no suco gástrico, já foi isolada em amostras de vômitos ${ }^{21}$. Esta pesquisa encontrou que crianças que freqüentaram creche ou escola apresentaram risco cerca de seis vezes maior de adquirem infecção pela Helicobacter pylori do que aquelas que não freqüentaram, fato que pode ser atribuído aos contatos próximos entre indivíduos que convivem em conglomerados.

Os resultados obtidos indicaram também que a presença de soropositividade para a infecção pela Helicobacter pylori nas crianças dependeu da soroprevalência em suas mães. 0 risco de uma criança apresentar-se com a infecção foi 19 vezes superior quando sua mãe também se encontrava positiva. Verificou-se ainda, que não houve criança soropositiva com sua respectiva mãe negativa, reforçando a hipótese de que a infecção ocorra principalmente dos adultos às crianças, tanto pela prevalência maior em adultos como pela infecção ser adquirida predominantemente na infância. Da mesma forma, quando se analisou a associação entre a freqüência de cepas CagA entre as mães e seus filhos, encontrou-se significância estatística. Estes achados são semelhantes aos de outros trabalhos publicados que consideraram que as mães infectadas podem ser importantes fontes de contaminação ${ }^{2831}$, embora não descartem a possibilidade de outras fontes isoladas ou em comum estarem influenciando a prevalência intrafamiliar ${ }^{12} 1732$. Cepas com CagA têm sido associadas a um risco aumentado para desenvolver úlcera péptica e câncer gástrico ${ }^{1833}$. Desta forma, as mães infectadas pela Helicobacter pylori parecem representar um risco para determinar a infecção em seus filhos, confirmando a hipótese de transmissão intrafamiliar da Helicobacter pylor $i^{1423}$, indicando que o contato direto pessoa a pessoa seja um modo de transmissão mediante as rotas oral-oral, fecal-oral e/ou gastro-oral.

Medidas de prevenção contra a infecção pela Helicobacter pylori envolvendo aspectos sociais, econômicos e culturais no ambiente intrafamiliar, com prioridade às condições higiênicas e sanitárias, devem ser amplamente estimuladas e aplicadas pelos serviços de saúde pública, particularmente entre a população carente, onde a exposição aos fatores de risco favorece a aquisição da bactéria.

\section{REFERÊNCIAS}

1. Araújo-Filho I, Brandão-Neto J, Pinheiro LAM, Azevedo IM, Freire FHMA, Medeiros AC. Prevalence of Helicobacter pylori infection in advanced gastric carcinoma. Arquivos de Gastroenterologia 43:288-292, 2006.

2. Ayres M, Ayres JRM, Ayres DL. BioEstat 5.0: aplicações estatísticas nas áreas das ciências biológicas e médicas. Belém: Sociedade Civil Mamirauá, $5^{\mathrm{a}}$ edição, Conselho Nacional de Desenvolvimento Científico e Tecnológico, Brasília, 2007.

3. Braden B, Caspary WF. Detection of Helicobacter pylori infection: when to perform which test? Annales Medicinae 33:91-97, 2001.

4. Brown LM. Helicobacter pylori: epidemiology and rotes of transmission. Epidemiologic Reviews 22:283-297, 2000.

5. Bruce MG, Maaroos HI. Epidemiology of Helicobacter pylori infection. Helicobacter 13: 1-6, 2008.

6. Bunn JEG, Mackay WG, Thomas JE, Reid DC, Weaver LT. Detection of Helicobacter pylori DNA in drinking water biofilms: implications for transmission in early life. Letters in Applied Microbiology 34:450-454, 2002.

7. Cammarota G, Tursi A, Montalto M, Papa A, Veneto G, Bernardi S, Boari A, Colizzi V, Fedeli G. Role of dental plaque in the transmission of Helicobacter pylori. Journal of Clinical Gastroenterology 22:174-177, 1996.

8. Censini S, Lange C, Xiang Z, Crabtree JE, Guiara P, Borodovsky M, Rapuolli R, Covacci A. Cag, a pathogenicity island of Helicobacter pylori, encodes type I-specific and disease-associated virulence factors. Proceedings of National Academy of Sciences of the United States 93:14648-14653, 1996.

9. Covacci A, Telford J, Giudice G., Parsonnet J, Rappuoli R. Helicobacter pylori virulence and genetic geography. Science 284:1328-1333, 1999.

10. Cover T, Blaser M. Helicobacter pylori factors associated with disease. Gastroenterology 117:257-260, 1999.

11. Deltenre M, Koster E. How come I've got it? European Journal of Gastroenterology \& Hepatology 12:479-482, 2000.

12. Dominice P, Bellentani S, Biasi AR, Saccoccio G., Rose A, Masutti F, Viola L, Balli F, Tiribelli C, Grilli R, Fusillo M, Grossi E. Familial clustering of Helicobacter pylori infection: population based study. British Medical Journal 319:537-541, 1999.

13. Dowsett SA, Archila A, Segreto VA, Gonzalez CR, Silva A, Vastola KA, Bartizek RD, Kowolik MJ. Helicobacter pylori in indigenous families of Central America: serostatus and oral and fingernail carriage. Journal of Clinical Microbiology $37: 2456-2460,1999$ 
14. Escobar ML, Kawakami E. Evidence of mother-child transmission of Helicobacter pylori infection. Arquivos de Gastroenterologia 41:239-244, 2004.

15. Fujimoto Y, Furusyo N, Toyoda K, Takeoka H, Sawayama Y, Hayashi J. Intrafamilial transmission of Helicobacter pylori among the population of endemic areas in Japan. Helicobacter 12:170-176, 2007.

16. Goodman JK, Correa P, Aux HJT, Ramírez H, Delany JP, Pepinosa OG. Helicobacter pylori infection in the Colombian Andes: a population-based study of transmission pathways. The American Journal of Epidemiology 144:144-290, 1996.

17. Han S, Zschausch HE, Meyer HW, Schneider T, Loos M, Bhakdi S, Maeurer MJ. Helicobacter pylori: clonal population structure and restricted transmission within families revealed by molecular typing. Journal of Clinical Microbiology 38:3646-3651, 2000.

18. Kato S, Sugivama T, Kudo M, Ohnuma K, Ozawa K, linuma K, Osaka M, Blaser M. CagA antibodies in Japanese children with nodular gastritis or peptic ulcer disease. Journal of Clinical Microbiology 38:68-70, 2000

19. Kikuchi S, Kurosawa M, Sakiyama T. Helicobacter pylori risk associated with sibship size and family history of gastric diseases in Japanese adults. Cancer Science 89:1109-1112, 2005.

20. Lehours P, Yilmaz 0. Epidemiology of Helicobacter pylori infection. Helicobacter 12:1-3, 2007.

21. Leung WK, Siu KLK, Kwok CKL, Chan SY, Sung R, Sung JJY. Isolation of Helicobacter pylori from vomitus in children and its implication in gastro-oral transmission. The American Journal of Gastroenterology 94:2881-2884, 2004.

22. Nabwera HM, Tam JSN, Logan RFA, Logan RPH. Prevalence of Helicobacter pylori infection in Kenyan schoolchildren aged 3-15 years and risk factors for infection. European Journal of Gastroenterology \& Hepatology 12:483-487, 2000.

23. Ng BL, Ng HC, Goh KT, Ho B. Helicobacter pylori in familial clusters based on antibody profile. Immunology and Medical Microbiology 30:139-142, 2006.

24. Oderba G., Rapa A, Marinello D, Ronchi B, Zavallone A. Usefulness of Helicobacter pylori stool antigen test to monitor response to eradication treatment in children. Alimentary Pharmacology Therapeutics 15:203-206, 2001.
25. Oliveira AM, Queiroz DMM, Rocha GA, Mendes EN. Seroprevalence of Helicobacter pylori infection in children of low socioeconomic level in Belo Horizonte, Brazil. American Journal of Gastroenterology 89:2201-2204, 1994.

26. Ozen A, Ertem D, Pehlivanoglu E. Natural history and symptomatology of Helicobacter pylori in childhood and factors determining the epidemiology of infection. Journal of Pediatric Gastroenterology and Nutricion 42:398-404, 2006.

27. Parsonnet J, Shmuely H, Haggerty T. Excreção fecal e oral de Helicobacter pylori por adultos sadios infectados. Journal of American Medical Association - Brasil 4:2935-2944, 2000

28. Queiroz DM, Mendes EN, Carvalho AS, Rocha G.A, Oliveira AM, Soares TF, Santos A, Cabral MM, Nogueira AM. Factors associated with Helicobacter pylori infection by a cagA-positive strain in children. The Journal of Infectious Diseases 181:626$630,2000$.

29. Rodrigues MN, Queiroz DMM, RT, Rocha AMC, Luz CRL, Braga LBC. Prevalence of Helicobacter pylori infection in Fortaleza, Northeastern Brazil. Journal of Public Health 39:847-849, 2005.

30. Rodrigues RV, Corvelo TC, Ferrer MT. Seroprevalence of Helicobacter pylori infection among children of different socioeconomic levels in Porto Velho, state of Rondônia. Revista Brasileira de Medicina Tropical 40:550-554, 2007.

31. Shimizu T, Yarita Y, Kaneko K, Yamashiro Y, Segawa 0, Ohkura R, Taneike I, Yamamoto T. Case of intrafamilial Helicobacter pylori reinfection after successful eradication therapy. The Pediatric Infectious Disease Journal 19:901-903, 2000.

32. Siavoshi F, Salmanian AH, Kbari FA, Malekzadeh R, Massarrat, S. Detection of Helicobacter pylori-specific genes in the oral yeast. Helicobacter 10:318-322, 2005.

33. Vandenplas Y, Blecker U. Helicobacter pylori infection in children. Acta Paediatrica 87:1105-1112, 2007.

34. Yañez P, Garza AM, Pérez-Pérez G., Cabrera L, Muñoz, 0, Torres, J. Comparison of invasive and noninvasive methods for the diagnosis and evaluation of erradication of Helicobacter pylori infection in children. Archives of Medical Research $31: 415-421,2000$ 